\title{
Índices de anillos de crecimiento en dos coníferas del Centro de México
}

\section{Growth ring indices in two conifers from central México}

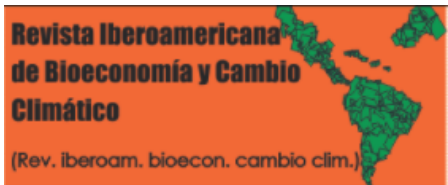

\author{
Gómez-Guerrero, Armando; Martínez-Molina, Gabriel; Martínez- \\ Trinidad, Tomás; Velázquez-Martínez, Alejandro; Sardiñas Gómez, \\ Oreste; Rivera, Carlos; Toruño, Pedro José; Editor Académico Prof. Dr. \\ Carlos A. Zuniga González
}

\author{
Armando Gómez-Guerrero \\ agomezg@colpos.mx \\ Colegio de Postgraduados-Campus Montecillo. \\ México, Mexico \\ Gabriel Martínez-Molina \\ Colegio de Postgraduados Campus Montecillo. \\ México, Mexico \\ Tomás Martínez-Trinidad \\ Colegio de Postgraduados Campus Montecillo. \\ Mexico, Mexico \\ Alejandro Velázquez-Martínez \\ Colegio de Postgraduados Campus Montecillo. \\ Mexico, Mexico \\ Oreste Sardiñas Gómez \\ orestess@geotech.cu \\ Instituto de Geografía Tropical, Cuba, Cuba \\ Carlos Rivera \\ crivera20@gmail.com \\ CURLA- UNAH, Honduras, Honduras
}

iD Pedro José Toruño

pjoseto@gmail.com

Universidad Nacional Autónoma de Nicaragua, León.,

Nicaragua

Editor Académico Prof. Dr. Carlos A. Zuniga

González

Universidad Nacional Autónoma de Nicaragua, León,

Nicaragua

Revista Iberoamericana de Bioeconomía y Cambio Climático

Universidad Nacional Autónoma de Nicaragua, León, Nicaragua ISSN-e: 2410-7980

Periodicidad: Semestral

vol. 1 , núm. 1,2015

czuniga@ct.unanleon.edu.ni

Recepción: 20 Agosto 2014

Aprobación: 15 Noviembre 2014
Resumen: Se determinaron índices de anillo de crecimiento (IAC) en Abies regiogiosa (Kunth Schltdl. et Cham) (oyamel) y Pinus hartwegii (Lindl.) (pino) en un transecto sobre el Eje Neovolcánico Transversal, en México. La toma de muestras de anillos se realizó en seis montañas que presenan condiciones climáticas, geología, edafología suelo y vegetación similares. Las montañas son: Nevado de Colima, Pico de Tancítaro, Nevado de Toluca, Cerro Tláloc, La Malinche y Pico de Orizaba. Se colectaron virutas de incremento de al menos 20 árboles longevos de cada especie en cada sitio; sin embargo, para fines de este estudio se seleccionaron los árboles más representativos quedando entre 6 y 17 árboles de cada especie en cada montaña (144 árboles en total). Los resultados mostraron tendencias diferentes entre especies y montañas. Sin embargo, estimando valores promedio de IAC para el transecto, se observa que en el año 1935 hubo una reducción de crecimiento para ambas especies. El Oyamel mostró mayor variación en los IAC. De las dos especies, sólo en Oyamel se observó relación significativa entre los índices de anillo y la precipitación.

Abstract: Tree ring indices (TRI) were determined in Abies Religiosa (Kunth Schltdl. et Cham) (fir) and Pinus Hartwegii (Lindl.) (pine) along a transect on the Neovolcanic axis, in Mexico. Wood core were sampled at six mountains with similar climatic, geological, soil and vegetation conditions. The mountains were: Nevado de Colima, Pico de Tancitaro, Nevado de Toluca, Cerro Tlaloc, La Malinche and Pico de Orizaba. Increment cores were collected from at least 20 long-lived trees from each species in each site; however, at the end of this study the most representative trees were selected leaving in between 6 to 17 trees of each species in each mountain ( 144 trees in total). The results indicated different trend of TRI for mountains and species. However, mean values for the whole transect showed that about the year 1935 there was a growth reduction for both species. Fir showed a greater variation TRI. Only fir data showed a significant relationship between ring indices and precipitation.

Keywords: Crecimiento radial, Bosques de alta elevación, Indicadores de clima, Radial growth, High altitude forests, Climate indicators. 
DOI: https://doi.org/10.5377/ribcc.v1i1.2146

Autor de correspondencia: agomezg@colpos.mx

\section{INTRODUCCIÓN}

Una característica de las coníferas es generar anillos anuales de crecimiento los cuales permiten relacionar la productividad forestal con variables climáticas, relacionar factores abióticos con el crecimiento, reconstruir el clima del pasado y generar indicadores de cambio climático (Klepac, 1983; Villanueva et al., 2008). Fisiológicamente, el ancho de anillo está en función del volumen o masa foliar de la copa, de la actividad de los reguladores de crecimiento y de la cantidad de productos de la fotosíntesis (Spurr y Barnes, 1982), pero son los factores ambientales los que determinan la intensidad de crecimiento y dejan su huella expresada en el ancho de anillo.

El uso de los anillos de crecimiento para el análisis de productividad en forma espacial y temporal de los bosques se ha empleado para conocer la relación entre ancho de anillo y condiciones meteorológicas (Robinson et al., 1990). Sin embargo, es importante señalar que el ancho de anillos está afectado por diversos factores entre los que destacan, edad del árbol, posición del árbol en la estructura vertical del rodal, factores bióticos (plagas) y factores abióticos (incendios). De tal manera que es muy importante considerar procesos de estandarización de los datos crudos de ancho de anillos de crecimiento y transformarlos a un indicador de anillo de crecimiento (IAC) el cual refleja fases de crecimiento de un grupo de árboles de diferente edad y con respecto a la media esperada de crecimiento (Cook y Briffa, 1990).

En este estudio se analizan IAC medidos sobre un transecto que se distribuye a lo largo del eje Volcánico Transversal de México (EVTM), considerando Abies religiosa (Kunth) Schltdl. \& Cham y Pinus hartwegii Lindl. en seis montañas del centro de México que son: Nevado de Colima (NC), Pico de Tancítaro (PT), Nevado de Toluca (NT), Cerro Tláloc (CT), La Malinche (MA) y Pico de Orizaba (PO). El índice de anillo de crecimiento se analizó por especie, por montaña y de manera global. Adicionalmente, se realizaron correlaciones entre el índice de anillo de crecimiento con la precipitación total y temperatura media anual. El objetivo principal fue analizar la tendencia en el ritmo de crecimiento radial de pino y oyamel con el fin de emplear los resultados de este estudio como una línea base con la que se puedan hacer comparaciones en el futuro y se pueda inferir posibles efectos de cambio climático sobre las masas forestales de las montañas del centro de México (Alien et al. 2002)

\section{MATERIALES Y MÉTODOS}

\section{Área de estudio}

El área de estudio corresponde a un transecto de seis montañas distribuidas a lo largo de $675 \mathrm{~km}$ sobre el Eje Neo volcánico Transversal de México (ENTM), donde existe poblaciones de Abies religiosa y Pinus hartwegii. Las montañas estudiadas tienen vegetación, clima y geología y suelos similares. Las altitudes sobre el nivel del mar de las montañas seleccionadas son: Nevado de Colima 4330 m, Cerro 4160 m, Nevado de Toluca 4560 m, Tláloc 4150 m, La Malinche 4460 m, y Pico de Orizaba 5650 m (Rzedowski, 1981) (Figura 1).

\section{NotAS DE AUTOR}




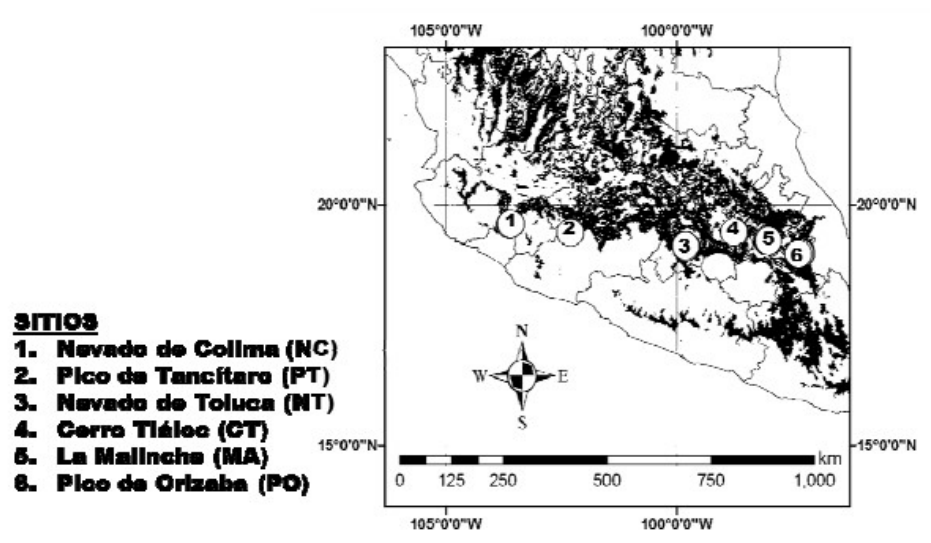

FIGURA 1.

Ubicación de los sitios de estudio

Elaboración propia

\section{Muestreo de virutas}

En cada montaña se hizo un recorrido para conocer las condiciones del lugar y localizar los árboles representativos de ejemplares de Abies religiosa y Pinus hartwegii. Para cada especie se eligieron entre $10 \mathrm{y}$ 20 árboles sanos y en lo posible longevos considerando su forma de copa, separación de entrenudos, altura, y diámetro. El número de árboles por sitio dependió de la localización de sitios adecuados para el muestreo y de las condiciones de seguridad para el acceso a cada lugar. Después de la selección de árboles adecuados en laboratorio, el número de individuos por sitios varió de 7 a 18 por sitio y para cada especie. Las virutas para el análisis de anillos de crecimiento se obtuvieron con un taladro de Pressler marca HAGLOF, modelo BS009 de $12 \mathrm{~mm}$ de diámetro interno. La viruta se tomó a $1.30 \mathrm{~m}$ (DAP) sobre el nivel del suelo y en cada sitio de colecta se geo-referenció.

Las virutas se etiquetaron y se envolvieron en papel aluminio para su protección y traslado. En el laboratorio, las virutas se secaron a temperatura de ambiente por 5 días. Con la virutas secas se procedió a realizar un corte longitudinal con una sierra contorneadora marca RYOBI de sierra fina 5 " para obtener dos muestras con cara limpia y plana de los anillos. Los cortes longitudinales de las virutas se pulieron con una lija fina para mejorar el contraste de los anillos. La caras planas y pulidas de las virutas se escanearon y se midió en ancho de anillos usando el paquete de cómputo llamado WINDENDRO.

Estandarización de la información de ancho de anillo

Para estandarizar los datos del ancho de anillos de crecimiento se procedió de acuerdo a Cook y Briffa (1990) y Villanueva et al. (2010). Para cada árbol se ajustó un modelo polinomial de orden 1 a 4 según se requiera para describir el ancho de anillo como función del tiempo. En algunos casos, se empleó el modelo exponencial. Una vez ajustado el modelo de regresión se estimó un valor predicho de anillo de crecimiento con base en el modelo de regresión. El índice de anillo de crecimiento (IAC) se estimó como el cociente del valor real del anillo y el estimado por la ecuación de regresión.

Para posicionar los valores de IAC en una media común se verificó que la distribución de los IAC tuviera media uno. En los casos que la media no fuera uno, se empleó la herramienta de Excel llamada "Solver" para recalcular los parámetros de la regresión de tal forma que la media de los IAC se ajustara a uno. Con este proceso de estandarización la comparación entre árboles de diferente edad y localidad fue válida.

Estandarización de la información de ancho de anillo

Se seleccionaron estaciones meteorológicas lo más cercano posibles a las montañas, para relacionar los IAC con la precipitación total, invernal (Enero a Abril) y de verano (Julio a Septiembre) y con la temperatura 
media anual. Sin embargo, no siempre fue posible encontrar disponibilidad de datos en las estaciones más cercanas, ya que en algunas de ellas se tienen datos incompletos y dejaron de funcionar desde los años $80 \mathrm{y}$ 90. Por lo anterior, dentro de lo posible se buscaron de una a dos estaciones cercanas a los sitios y se analizó la correlación entre IAC y variables climáticas.

Se extrajo la información de la base de datos conocida como Eric III versión 2. Las estaciones elegidas para NC, PT, NT, CT, MA y PO fueron respectivamente: Quito, Jalisco y Tecomán, Peribán, Michoacán, Nevado de Toluca, Estado de México, Rió Frío Estado de México y Ávila Camacho, Achichapa, Tlaxcala y Tetelzingo, Veracruz.

\section{Análisis estadístico}

Se estimaron IAC por montaña y especies y para el transecto completo. Se graficaron las tendencias del IAC en el tiempo y sus indicadores de dispersión. Se hicieron análisis de regresión entre IAC y variables climáticas para cada sitio y por especie para seleccionar las correlaciones estadísticamente significativas.

\section{Resultados Y Discusión}

\section{Índices de anillo de crecimiento por especie y montaña}

En oyamel, los resultados muestran que en general hubo una fase de bajo crecimiento cercano al año 1925 (Figura 2). Posterior a este año hubo recuperación del crecimiento pero nuevamente cercano a 1975 hubo otra fase de bajo crecimiento. Esta tendencia es principalmente en el Nevado de Colima, Nevado de Toluca, La Malinche y Pico de Orizaba. No se observó esta tendencia en Pico de Tancítaro; lo cual indica diferencias en el ritmo de crecimiento de oyamel entre sitios. Los ejemplares más viejos se muestrearon en Nevado de Colima y Pico de Orizaba. Los individuos más jóvenes se colectaron en Nevado de y Cerro Tláloc.

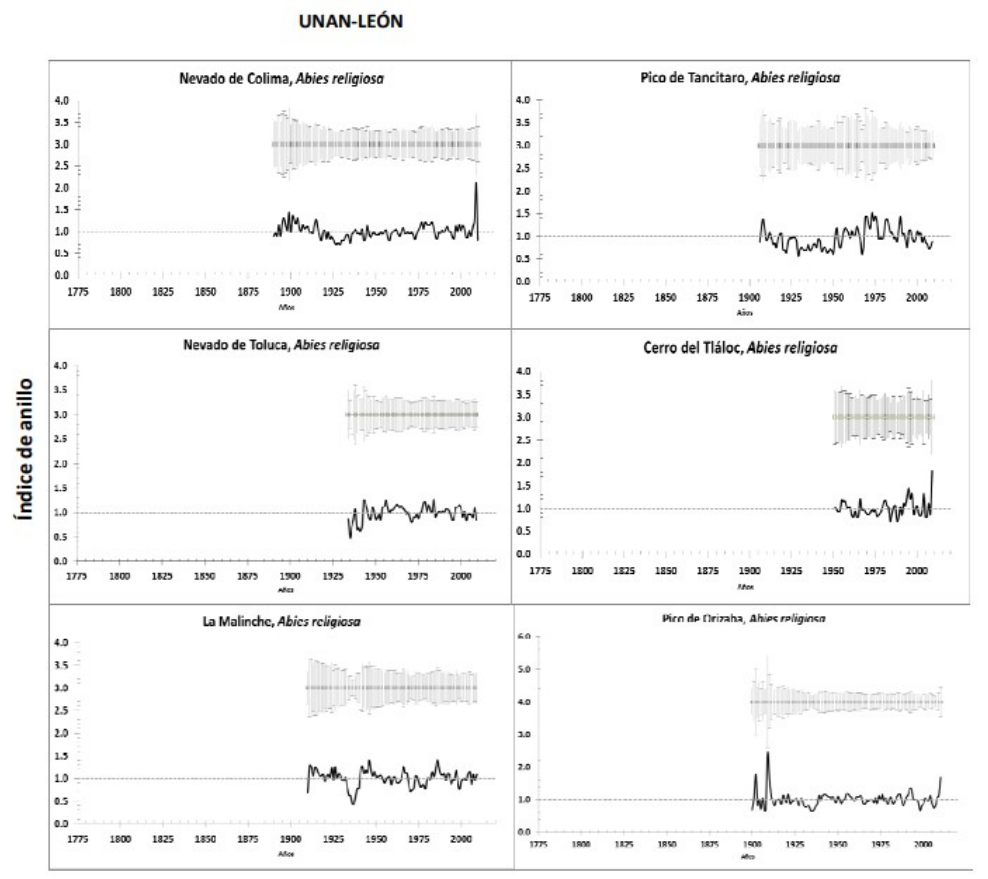

FIGURA 2.

Índices de anillo de crecimiento para Abies religiosa. Se indica el error estándar en la parte superior. Elaboración propia

En el caso de pino, en el nevado de Colima se refleja bajo crecimiento del 1825 a1860. No se observan eventos comunes de alto o bajo crecimiento en el resto de las montañas. A diferencia de oyamel, en pino no se detecta el efecto de la erupción del Volcán de Colima de 1913 (Biondi et al., 2003) indicando mayor 
susceptibilidad a impactos ambientales en los bosques de oyamel (Harteau et al. 2007). Existe una tendencia de mayor crecimiento de pino en los últimos años en Nevado de Toluca y Pico de Orizaba una tendencia similar a la que sugieren McMahon et al. (2010) (Figura 3). Sin embargo, se requiere un estudio a mayor detalle para saber si esta tendencia no está afectada por arbolado viejo dentro de la muestra que puede influir en sobre estimaciones de crecimiento (Nehrbass - Ahles et al., 2014). Un crecimiento mayor al esperado en los últimos años se podría explicar diversos factores como un efecto de fertilización por CO2 o una influencia en la eficiencia de uso de agua de las especies forestales (Silva et al., 2009).

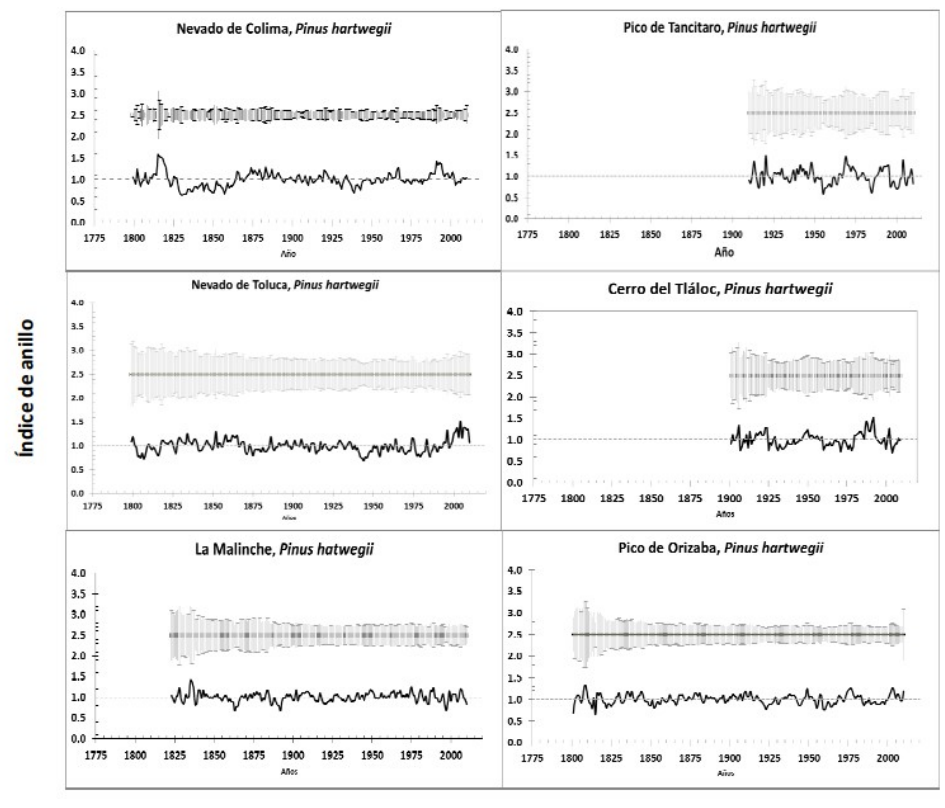

FIGURA 3.

Índices de anillo de crecimiento para Abies religiosa. Se indica el error estándar en la parte superior.

Elaboración propia

\section{Índices de anillo de crecimiento por especie}

Los resultados mostraron que Pinus hartwegii es una especie más longeva debido a que se encontraron registros desde el año 1,750 a la fecha, mientras que Abies religiosa presentó individuos 100 años más jóvenes con registros desde el año 1850 (Figura 4). La variación de IAC es más amplia en oyamel comparada con pino, indicando que oyamel es más sensible a cambios ambientales. En oyamel también se destaca una disminución de crecimiento en 1935, la cual también fue detectada por Alarcón (1993).

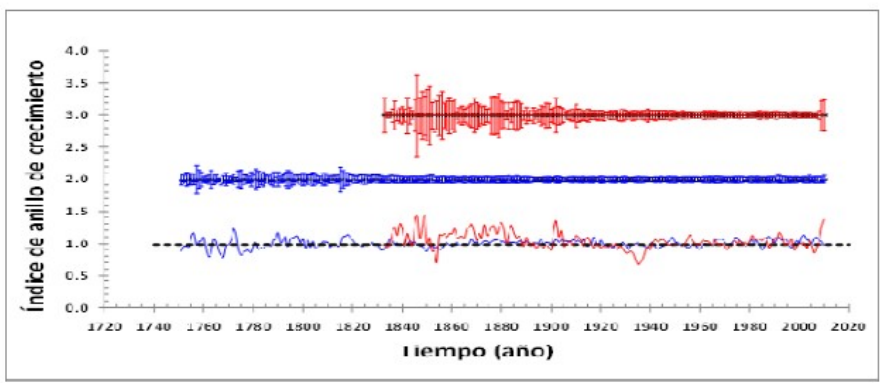

FIGURA 4.

Índices de anillo de crecimiento para Abies religiosa y

Pinus hartwegii en seis montañas del centro de México

Elaboración propia 


\section{Índices de anillo de crecimiento y variables meteorológicas}

No se encontró correlación entre los IAC y la temperatura media anual. Oyamel fue la única especie que muestra cierto grado de correlación con la precipitación total, de verano e invierno. Pero los resultados varían en cada localidad. Con En Colima los datos de observación fueron de 1953 a 2008 y no se encontró correlación significativa con precipitación dentro de dicho periodo. En CT el periodo de datos fue de 1980 a 1997, y sólo en el periodo 1992 a 1997 se encontró una correlación de 0.67. En NT el periodo de datos fue de 1969 a 1986; pero sólo en el periodo 1974 a 1986 se encontró una correlación de 0.42. En CT el periodo fue de 1970 a 1980, pero hubo correlación sólo en el periodo 1977 a 1980 con una correlación de 0.52. En MA el periodo observado fue de 1967 a 1976 y no se muestra correlación. Finalmente, en PO, el periodo de observación de 1967 a 1986 y en el periodo de 1971 a 1978 se encontró una correlación de 0.71 . Las relaciones más importantes entre IAC y variables climáticas fueron con la lluvia invernal y de veranos. Los resultados indican que la lluvia invernal debe ser de al menos de $100 \mathrm{~mm}$ para mantener los ritmos de crecimiento esperados en oyamel y la precipitación de verano debe ser de $850 \mathrm{~mm}$. Las precipitaciones menores o mayores a estos valores resultarán en bajo o altos crecimiento. Este es un resultado relevante del estudio que puede utilizarse como la precipitación crítica para predecir efectos de cambio climático en bosques de oyamel (Figura 5).

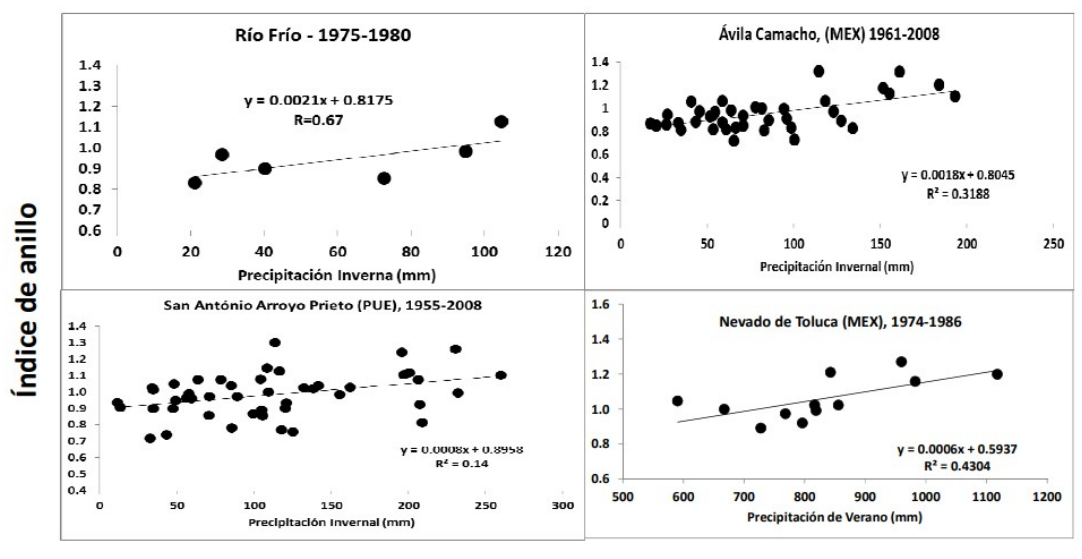

FIGURA 5

Relación del índice de anillo de crecimiento en Abies religiosa y la precipitación invernal y de verano, calculada en cuatro estaciones diferentes Elaboración propia

Es posible que sólo se haya encontrado correlación entre precipitación y crecimiento de oyamel por tratarse de una especie que demanda mucha agua y es más sensible a las sequias. Estos resultados sugieren que para evaluar efectos de cambio climático en el corto plazo, Abies religiosa es una especie más adecuada, mientras que para periodos más largos, Pinus hartewgii es la especie conveniente. Harteau et al. (2007), también encontró que para fines de estudios del clima Abies concolor en comparación con Pinus jeffreyi, sugierindo que la raíz de pino podría explorar mayor profundidad de suelo y abastecerse de agua en periodos secos (Stephens et al. 2003).

Comparando los resultados de este trabajo con los de Diaz et al. (2001) destaca la importancia de contar con bases climáticas cercanas a las áreas de estudio. Desafortunadamente las bases climáticas en México son limitadas para establecer relaciones significativas entre los anillos de crecimiento y el clima. Arreóla-Ortíz y Navar-Cháidez (2010) si encontraron una relación entre el ancho de anillos de Pseudotsuga menziesii con la precipitación. Esta relación podría explicarse por una relación similar a la de oyamel ya que también es una especie con alta demanda de agua. Los anillos de crecimiento en Pseudotsuga menziesii se definen con mayor claridad (Constante et al., 2010) en comparación con otras especies de coníferas, lo que también puede ayudar a incrementar la precisión en las mediciones y por ende mejorando la relación con variables 
climáticas (Villanueva et al., 2008). La relación entre el ancho de anillo y variables climáticas se obscurece por la influencia de otros factores y en algunos estudios las relaciones no explican más de $28 \%$ de la variación entre anillos y clima (Trouet et., 2010).

\section{Conclusiones}

La tendencia de los índices de anillo de crecimiento fue diferente entre Pinus hartwegii y Abies religiosa. Existe una mayor variación en los índices de crecimiento de oyamel, comparados con los de pino. Además, se observó que aproximadamente en el año 1935 hubo un bajo crecimiento para ambas especies; lo cual puede ser una indicación de la afectación por algún factor ambiental. Cada montaña muestra un patrón específico de crecimiento en anillos. Oyamel mostró mayor variación en los índices de crecimiento y un incremento en los últimos 3 años. En el caso de pino, sólo en las montañas del Nevado de Toluca y Pico de Orizaba se observó una tendencia de incremento en crecimiento del año 2000 a la fecha. De las dos especies, sólo en Oyamel se observó relación entre los índices de anillo y la precipitación total, lo que puede deberse a la sensibilidad de la especie en los cambios de humedad del suelo.

\section{REFERENCIAS}

Allen, CD., Savage, M., Falk, DA., Suckling, KF., Swetnam, TW., Schulke PB., Stacey p., Morgan P., Huffman M. y Klingel, JT. (2002). Restauración ecológica de los ecosistemas de pino ponderosa del suroeste: una perspectiva amplia. Aplicaciones ecológicas , 12 (5), 1418-1433.

Alarcón, M C. (1993). Estudio dendrocronológico de Pinus hartwegii Lindl en el Suroeste de la cuenca de México. Tesis de Maestría. Colegio de Postgraduados. México.

Arreóla-Ortíz, M R Y Návar-Cháidez J J. (2010). Análisis de sequías y productividad con cronologías de Pseudotsuga menziesii Rob. \& Fern y su asociación con El Niño en el Noreste de México. Boletín de Instituto de Geográfía de la UNAM 71: 7-20.

Biondi F; Estrada-Galindo I., Gavilanes-Ruiz JC., Elizalde-Torres A. (2003). Tree growth response to the 1913 eruption of Volcan de Fuego de Colima, Mexico. Quaternary Research 59: 293-299.

Constante, V G,. Villanueva J., Cerano J. y Estrada J. (2010). Parámetros para definir el potencial dendrocronólogico. Folleto Técnico 19. Instituto Nacional de Investigaciones Forestales, Agrícolas y Pecuarias. México. 40p.

Cook E. y Briffa k. (1990). Data analysis. In: E. R. Cook and L. A. Kairiukstis (Eds.). Methods of dendrochronology: Applications in the environmental sciences. Kluwer Academic Publisher. Netherlands. p. 92-162.

Díaz, SC,. Touchan, R. y Swetnam, TW. (2001). Una reconstrucción de anillos de árboles de precipitaciones pasadas para Baja California Sur, México. Revista internacional de climatología: una revista de la Royal Meteorological Society , 21 (8), 1007-1019.

Hurteau, M., Zald, H. y North, M. (2007). Species-Specific response to climate reconstruction in upper- elevation mixed-conifers of the Western Sierra Nevada, California. Can. J. For. Res. , 37 (9), 1681-1691.

Klepac D. (1983). Crecimiento e incremento de árboles y masas forestales. Segunda edición.

Nehrbass - Ahles, C., Babst, F., Klesse, S., Nötzli, M., Bouriaud, O., Neukom, R., Dobbertin M. \& Frank, D. (2014). The influence of sampling design on tree - ring - based quantification of forest growth. Global change biology, 20(9), 2867-2885.

Robinson, W. J., Cook, E., Pilcher, J. R., Eckstein, D., Kairiukstis, L., Shiyatov, S., \& Norton, D. A. (1990). Some historical background on dendrochronology. In Methods of Dendrochronology (pp. 1-21). Springer, Dordrecht.

Rzedowski, J. (1981). Vegetación de México. Primera Edición. Editorial Limusa. Mexico. 432 p. 
Silva, L. C., Anand, M., Oliveira, J. M., \& Pillar, V. D. (2009). Past century changes in Araucaria angustifolia (Bertol.) Kuntze water use efficiency and growth in forest and grassland ecosystems of southern Brazil: implications for forest expansion. Global Change Biology, 15(10), 2387-2396.

Spurr, S H. y Barnes B. V. (1982). Ecología forestal. Tercera Edición. AGT editor. S.A. Mexico. 690 p.

Stephens, S L., Skinner C. N., Gill S. J. (2003). Dendrochronology-based fire history of Jeffrey pine: Mixed conifer forests in the Sierra San Pedro Martir, Mexico. Can. J. For. Res. 33: 1090-1101.

Trouet, V., Esper J., Beckma H. (2010). Climate/growth relationships of Brachystegiaspiciformis from themiombo woodland insouthcentral Africa. Dendrochronologia 28:161-171.

McMahon, S. M., Parker, G. G., \& Miller, D. R. (2010). Evidence for a recent increase in forest growth. Proceedings of the National Academy of Sciences, 107(8), 3611-3615.

Villanueva, D J., Cerano J., Stahle D. W., Constante V., Estrada A. (2010). Estandarización y desarrollo de series dendrocronológicas en México. Folleto técnico 16. Instituto Nacional de Investigaciones Forestales, Agrícolas y Pecuarias. México. 55

Villanueva, J. D., J. Cerano P., D. W. Stahle, J. E estrada A. y V. Constante G. (2008). Potencial dendrocronológico de Pseudotsuga menzieii (Mirb.) Franco y reconstrucciones de precipitación y flujo en México. Folleto científico 23. Instituto Nacional de Investigaciones Forestales, Agrícolas y Pecuarias. México. 49 p. 